
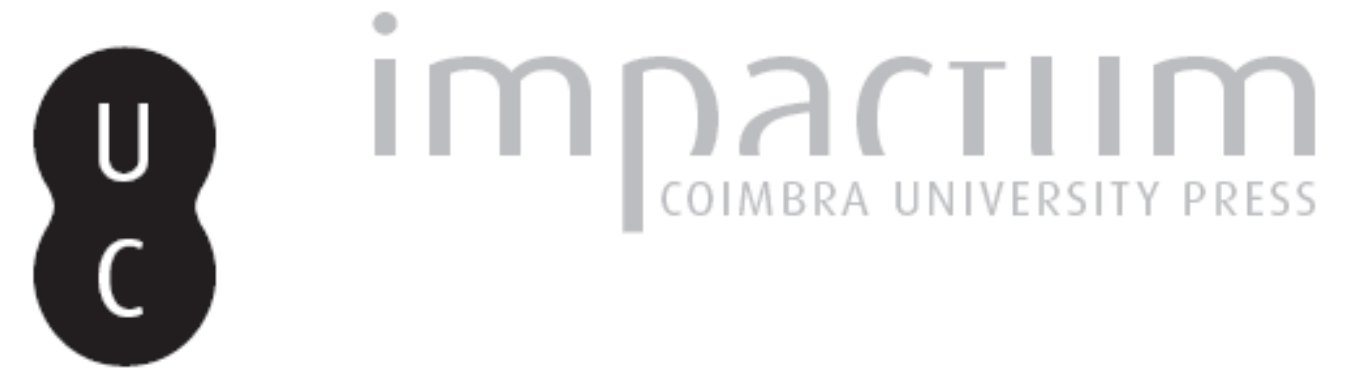

\title{
Estudo do risco sísmico e de tsunamis do Algarve (ERST)
}

Autor(es): $\quad$ Costa, Elsa; Vicêncio, Henrique; Pires, Patrícia

Publicado por: Associação Portuguesa de Riscos, Prevenção e Segurança

URL persistente:

URI:http://hdl.handle.net/10316.2/35858

DOI:

DOI:http://dx.doi.org/10.14195/1647-7723_19_6

Accessed : $\quad$ 26-Apr-2023 08:38:59

A navegação consulta e descarregamento dos títulos inseridos nas Bibliotecas Digitais UC Digitalis, UC Pombalina e UC Impactum, pressupõem a aceitação plena e sem reservas dos Termos e Condições de Uso destas Bibliotecas Digitais, disponíveis em https://digitalis.uc.pt/pt-pt/termos.

Conforme exposto nos referidos Termos e Condições de Uso, o descarregamento de títulos de acesso restrito requer uma licença válida de autorização devendo o utilizador aceder ao(s) documento(s) a partir de um endereço de IP da instituição detentora da supramencionada licença.

Ao utilizador é apenas permitido o descarregamento para uso pessoal, pelo que o emprego do(s) título(s) descarregado(s) para outro fim, designadamente comercial, carece de autorização do respetivo autor ou editor da obra.

Na medida em que todas as obras da UC Digitalis se encontram protegidas pelo Código do Direito de Autor e Direitos Conexos e demais legislação aplicável, toda a cópia, parcial ou total, deste documento, nos casos em que é legalmente admitida, deverá conter ou fazer-se acompanhar por este aviso.

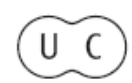




\section{territorium}

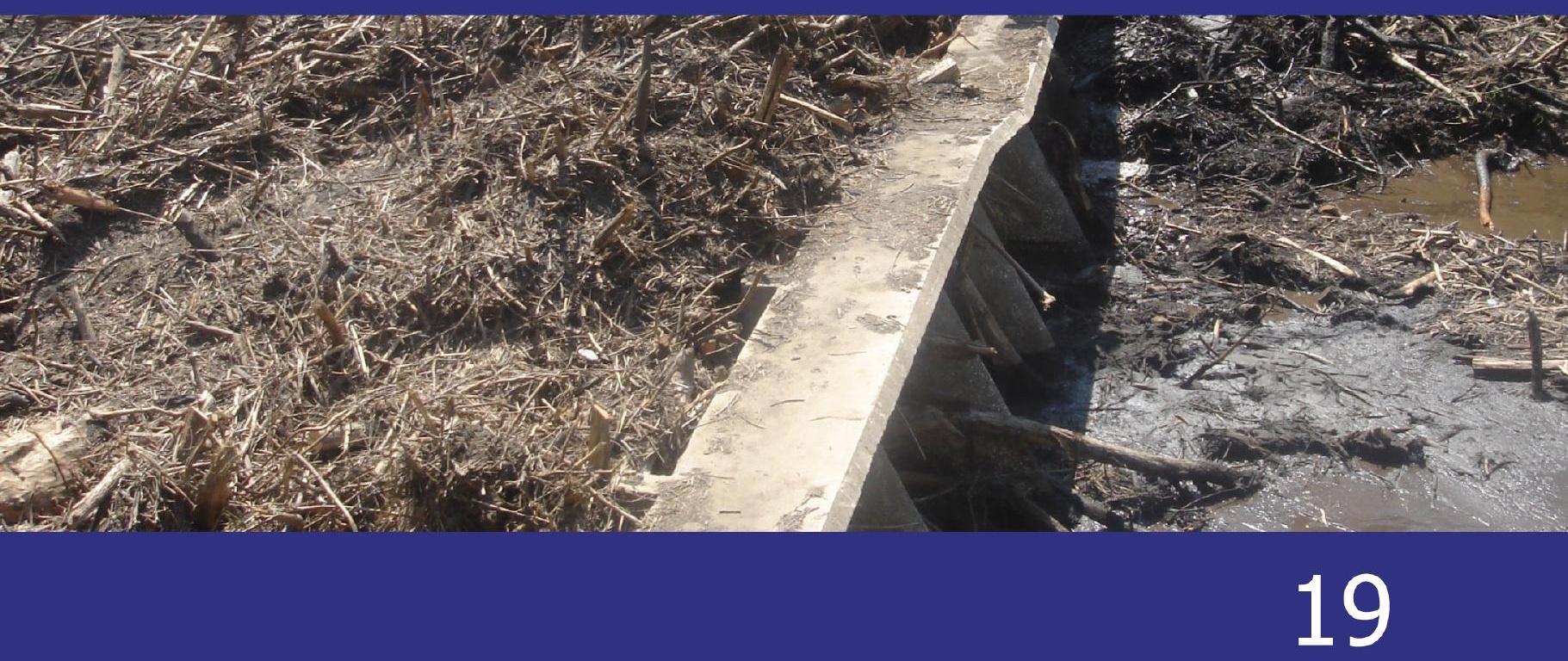

\section{Reequacionar o Conhecimento dos Riscos e das Catástrofes}

Revista da Associação Portuguesa de Riscos, Prevenção e Segurança 
Elsa Costa

Autoridade Nacional de Protecção Civil elsa.costa@prociv.pt

Henrique Vicêncio

Autoridade Nacional de Protecção Civil henrique.vicencio@prociv.pt

Patrícia Pires

Autoridade Nacional de Protecção Civil patrícia.pires@prociv.pt

\section{RESUMO}

O Estudo do Risco Sísmico e de Tsunamis do Algarve (ERSTA) teve como objectivo o conhecimento aprofundado do risco sísmico e de tsunamis na região e o desenvolvimento de um plano especial de emergência detalhado para estes riscos e, ainda, o desenvolvimento de políticas de prevenção e protecção adequadas para o Algarve. Com este Estudo pretendeu-se que todos os resultados técnico-científicos, que cobrem uma vasta gama de temas, desde a geração de sismos credíveis à estimativa dos danos dos elementos vulneráveis da sociedade, fossem implementados num simulador, que permitirá estimar e visualizar as previsões de danos, devidamente georreferenciadas.

Palavras chave: Risco sísmico, simulador, planeamento de emergência.

\section{RESUMEN}

El Estudio de Riesgo Sísmico y de Tsunami en el Algarve (ERSTA) - Dirigido para el conocimiento profundo de los riesgos sísmicos y de tsunamis en la región y para elaborar un plan de emergência detallado para estos riesgos y también el desarrollo de políticas de prevención y una protección adecuada en Algarve. Con este estudio se hay pretendido que todos los resultados técnicos y científicos, que abarcan una amplia gama de temas, desde la generación de los terremotos e estimación fiable de los daños a los elementos vulnerables de la sociedad, se llevaron a cabo en un simulador, que podrá estimar y ver las predicciones de daños, debidamente georeferenciados.

Palabras clave: Riesgo sísmico, simulador, planificación de emergencia.

\section{RÉSUMÉ}

L'étude du risque séismique et des risques de tsunamis dans l'Algarve (ERSTA) - En visant une connaissance approfondie du risque sismique et de tsunamis dans la région, l'élaboration d'un plan d'urgence détaillé pour ces risques et l'élaboration de politiques de prévention et d'une protection adéquate de l'Algarve. Avec cette étude, il était prévu que tous les résultats techniques et scientifiques, qui couvrent un large éventail de sujets, de la génération des tremblements de terre et estimation crédible des dommages aux éléments vulnérables de la société,ont été mis en oeuvre dans un simulateur, qui permettra d'évaluer et consulter les prévisions des dommages, bien géoréférencées.

Mots-clé: Risque sismique, simulateur, la planification d'urgence.

\section{ABSTRACT}

The Study of Seismic Risk and Tsunamis in the Algarve (ERSTA) - aimed the knowledge of seismic risk and tsunami in the region and the development of an emergency plan to these risks and also the development of prevention policies and adequate protection to the Algarve. With this study was intended that all technical and scientific results, which cover a wide range of topics from the generation of credible earthquakes to estimation of damage of the elements of society, are implemented in a simulator, which will estimate and view the predictions damage, properly georeferenced.

Key words: Seismic risk, simulator, emergency planning.

* O texto deste artigo corresponde à comunicação apresentada ao II Congresso Internacional de Riscos e VI Encontro Nacional, tendo sido submetido para revisão em 31-05-2010, tendo sido aceite para publicação em 29-07-2010.

Este artigo é parte integrante da Revista Territorium, n. ${ }^{\circ} 19,2012,{ }^{\circ}$ RIscos, ISBN: 0872- 8941. 


\section{Introdução}

Os registos históricos demonstram que a região do Algarve é a que, ao longo dos tempos, tem registado maiores intensidades sísmicas em Portugal Continental, sendo esta uma região de características particulares, pois além da grande concentração urbana junto ao litoral, recebe sazonalmente um intenso fluxo populacional, nacional e internacional.

Assim, considerando ainda a necessidade de se incorporar uma análise ao fenómeno e ao perigo de tsunamis que, mais do nunca, constituem uma ameaça real na região do Algarve, foi determinado pelo Estado que se concretizasse o Estudo do Risco Sísmico e de Tsunamis na Região do Algarve (ERSTA), de forma a dar cumprimento ao objectivo fundamental da protecção civil de prevenção dos riscos colectivos resultantes de acidentes graves ou catástrofes.

O Estudo do Risco Sísmico e de Tsunamis do Algarve abrangeu os 16 concelhos do distrito de Faro (Fig. 1), ao qual corresponde uma população presente diária máxima de cerca de 1,5 milhões de habitantes (GASPAR, 2008).

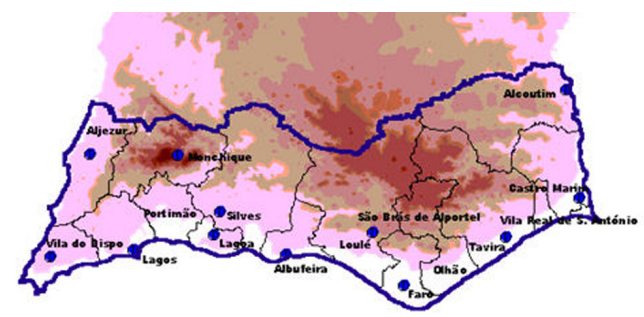

Fig. 1 - Área de estudo: concelhos da Região do Algarve. Fonte: ANPC, 2010.

\section{Características Gerais do Projecto}

O "Estudo do Risco Sísmico e dos Tsunamis do Algarve", designado daqui em diante como ERSTA, apresenta as seguintes Características Gerais do Projecto:

Início: Janeiro de 2007;

Duração: 2 anos;

Coordenação Geral: Autoridade Nacional de Protecção Civil (ANPC), apoiada por um coordenador científico;

\section{Equipas participantes:}

-Instituto das Ciências da Terra e do Espaço (ICTE/ FCUL);

- Instituto Superior Técnico (ICIST/IST);

-Laboratório Nacional de Engenharia Civil (LNEC);

- Universidade de Lisboa/ Faculdade de Letras/Centro de Estudos Geográficos (UL/FL/CEG);

- Universidade do Porto/Faculdade de Letras (FLUP);
- Universidade do Algarve/Escola Superior de Tecnologia (UALG/EST);

- Universidade do Algarve (UALG);

- Instituto Nacional de Engenharia, Tecnologia e Inovação (INETI);

- Instituto de Meteorologia (IM);

- Instituto Politécnico de Beja/Escola Superior de Tecnologia e Gestão/FUZZY (IPB/ESTIG/FUZZY).

O ERST contemplou as mais diversas componentes técnicocientíficas que permitem chegar à avaliação de danos quer humanos quer materiais provenientes de um qualquer cenário sísmico plausível. Este objectivo foi conseguido, juntando toda a informação num "Simulador de Cenários Sísmicos" que irá fundamentar o desenvolvimento de um Plano de Emergência para a região do Algarve. As principais vertentes abordadas foram (fig. 2):

\begin{tabular}{|ll|}
\hline Catálogo Sismico \\
\hline \hline Definição de Cenários e Caracterização da Energia Sísmica \\
\hline \hline Identificação dos elementos em risco e análise das vulnerabilidades \\
\hline \hline Avaliação dos danos \\
\hline \hline PLANEAMENTO DE EMERGÊNCIA/MITIGAÇÃO/ORDENAMENTO DO TERRITÓRIO \\
\hline \hline
\end{tabular}

Fig. 2 - Principais vertentes do ERSTA. Fonte: ANPC, 2010.

\section{O Simulador de Cenários e alguns Resultados}

Todos os dados coligidos, tratados e obtidos pelas equipas com representação espacial foram incluídos num Simulador de Cenários de forma georreferenciada (Mota de Sá, 2008). O Simulador, através dos algoritmos desenvolvidos pelas equipas, foi concebido por módulos que tratam as diferentes vertentes atrás mencionadas. O cálculo inicia-se pela escolha de um sismo, dando a Localização do Epicentro e Falha a ele associada, Magnitude, dia e hora da ocorrência (fig. 3). A partir daí, o Simulador calcula para cada unidade geográfica (Sub-Secção Estatística) um conjunto de parâmetros que exprimem a acção sísmica (com ou sem a influência dos Solos). Esta acção vai causar efeitos sobre os diferentes parques construídos, quantificando os danos ocorridos, e a população afectada.

O Simulador analisa em alguns módulos mais do que um modelo de trabalho, sendo os resultados apresentados em intervalos com limites superiores e inferiores. Os resultados estão expressos quer em mapas (georreferenciados) quer em tabelas que permitem o seu uso noutras utilizações.

Vamos seguidamente apresentar dois casos que ilustram as potencialidades do Simulador. 0 primeiro caso 


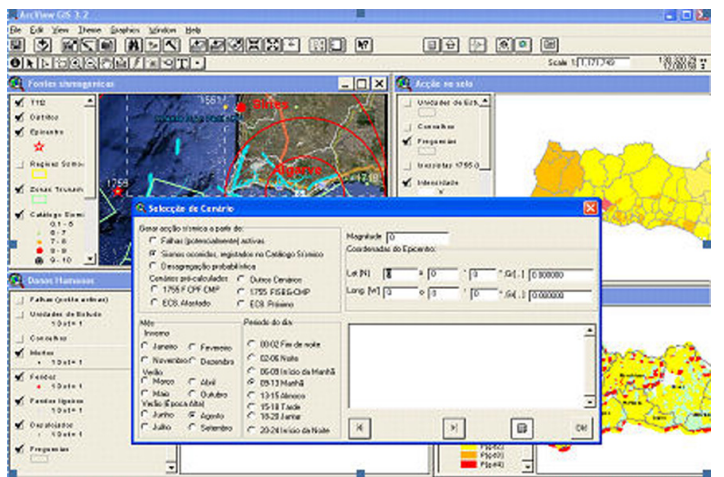

Fig. 3 - Painel de arranque do simulador. Fonte: ANPC, 2010.

(cenário A) é respeitante a um sismo com epicentro em terra (falha de Carcavai) e magnitude 6. O segundo caso corresponde ao epicentro do sismo ocorrido a 17 de Dezembro de 2009, com magnitude de 8.

Apresentam-se nas Figuras 4 a 6 e 7 a 9a carta de Intensidades, os danos no parque habitacional, e na população, respectivamente para os dois cenários.

\section{Cenário A}

-Epicentro: 13,8 Km, NNW de Faro (falha de Carcavai) - Magnitude: 6.0

-Data/Hora: Maio, 10:00 h
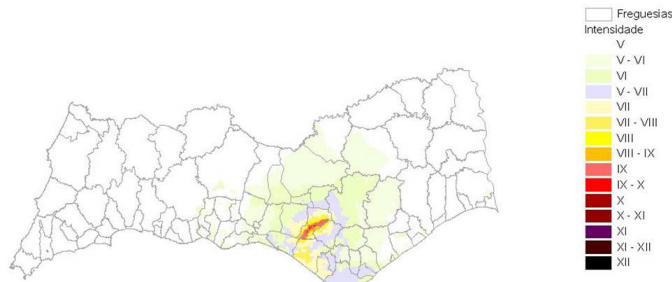

Fig. 4 - Carta de Intensidades EMS-98. Fonte: ANPC, 2010.

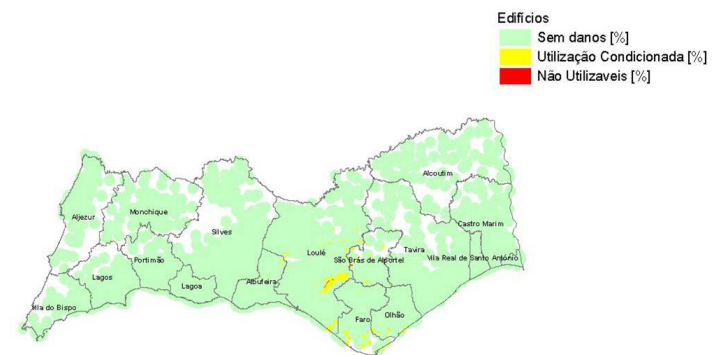

Fig. 5 - Danos no parque habitacional. Fonte: ANPC, 2010.

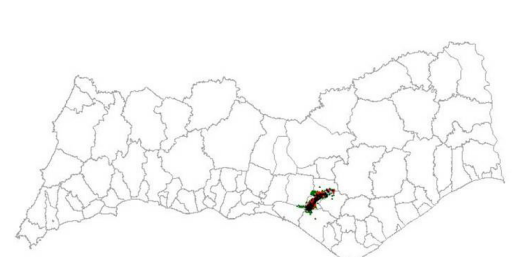

Mortos 1 Dot $=1$ Feridos 1 Dot $=1$ Feridos ligeiros Desalojados 1 Dot $=1$ Freguesias

Fig. 6 - Danos humanos . Fonte: ANPC, 2010.

\section{Cenário B}

-Epicentro: $188 \mathrm{Km}$, WSW de Faro

-Magnitude: 8.0

-Data/Hora: Agosto, 12:00h

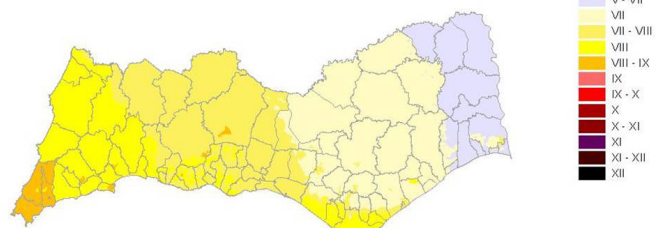

Fig. 7 - Carta de Intensidades EMS-98. Fonte: ANPC, 2010.

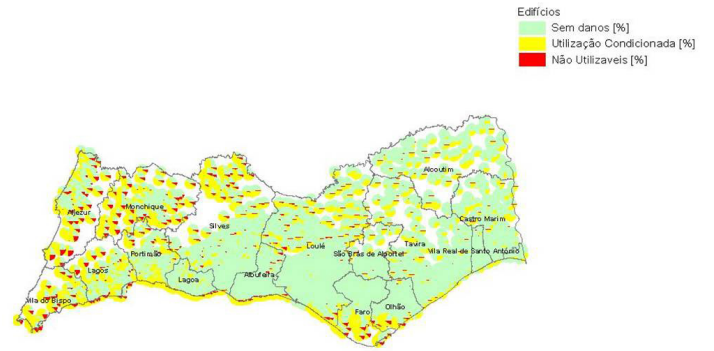

Fig. 8 - Danos no parque habitacional. Fonte: ANPC, 2010.

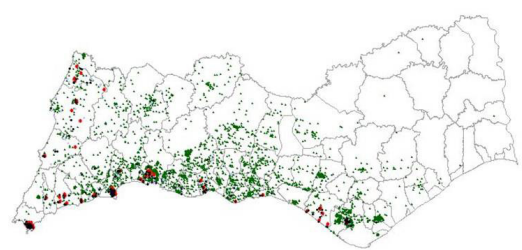

Mortos 1 Dot $=1$ Feridos . 1 Dot $=1$ Feridos ligeiros
1 Dot $=1$ Desalojados . 1 Dot $=1$ Freguesias

Fig. 9 - Danos humanos.

Na fig. 10 apresentam-se ainda as zonas inundadas na região da Quarteira e Vila Moura para o cenário B.

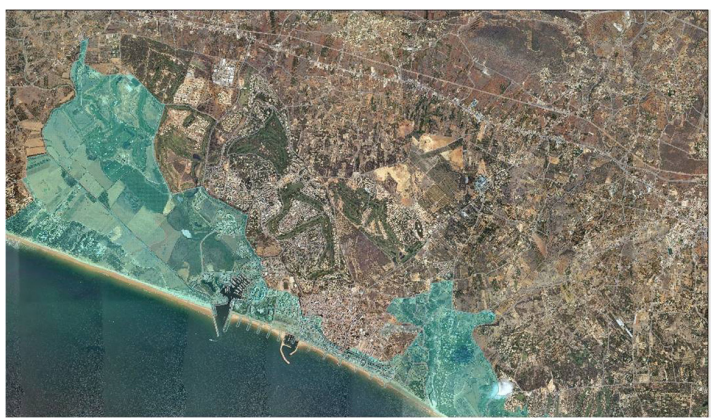

Fig. 10 - Zonas inundadas pelo Tsunami (Pormenor de Quarteira e Vilamoura) (BAPTISTA, 2008)

\section{Planeamento de Emergência}

O desenvolvimento de cenários sísmicos conduz a uma avaliação de danos que permite não só programar as acções de protecção civil antes da emergência, ao nível da adopção de medidas preventivas e de mitigação, mas também durante a emergência na definição das zonas de intervenção (Sistema Integrado de Operações de Protecção e Socorro) na qual assentará a organização da resposta dos agentes de protecção civil.

Após a fase de emergência, onde são criadas as condições e utilizados os meios indispensáveis à minimização de 
consequências, existe todo um conjunto de acções e medidas de recuperação da normalidade e prevenção de novos acidentes. Delas fazem parte, designadamente, as operações de limpeza de entulhos e as acções de inspecção que são necessárias para garantir as condições de utilização segura dos alojamentos afectados.

Seguidamente, apresentam-se alguns resultados que foi possível apurar, com base em estimativas de valores médios, quer do volume de entulhos a remover, quer do número de equipas de técnicos especializados para levar a cabo as inspecções para o universo dos edifícios que ficariam com utilização condicionada (em função do grau de dano (ver QuadRo I)).

QuADro I - Valores médios de danos humanos e no parque

\begin{tabular}{|l|c|c|}
\hline \multicolumn{1}{|c|}{ edificado. } \\
\hline Danos (valor médio) & Carcavai, 6 & $\begin{array}{c}\text { SW Cabo } \\
\text { S. Vicente, 8 }\end{array}$ \\
\hline Feridos ligeiros & 954 & 5.097 \\
\hline $\begin{array}{l}\text { Feridos necessitar } \\
\text { cuidados hospitalares }\end{array}$ & 46 & 520 \\
\hline Mortos & 51 & 178 \\
\hline
\end{tabular}

\begin{tabular}{|l|c|c|}
\hline \multicolumn{1}{|c|}{ Danos (valor médio) } & Carcavai, 6 & $\begin{array}{c}\text { SW Cabo } \\
\text { S. Vicente, 8 }\end{array}$ \\
\hline Edifícios colapsados & 280 & 782 \\
\hline Edifícos danos severos & 775 & 4.421 \\
\hline $\begin{array}{l}\text { Edifícios utilização } \\
\text { condicionada }\end{array}$ & 8.042 & 44.728 \\
\hline
\end{tabular}

Com base na caracterização do parque edificado desenvolvida no ERSTA (LNEC, 2008), foi possível apurar o número médio de pisos e de alojamentos com os quais se estimou de forma aproximada o volume de entulhos, assumindo uma área média de construção de $250 \mathrm{m2} /$ piso a que deverá corresponder um volume de construção de cerca de 1350 m3 (LOUREnço, 2007).

Para a estimativa do número de técnicos necessários para a realização das inspecções, considerou-se a experiência adquirida em Áquilla, Itália, por um elemento da ANPC. Assim, assumindo-se um período médio de 3 meses para duração das visitas técnicas, por equipas de 2 elementos, admitindo ainda a realização de 5 inspecções/dia, é possível verificar que, no cenário mais gravoso, seriam necessários mais de 300 técnicos a actuar no terreno (ver QuADro II).

\section{Conclusões}

Com base no simulador desenvolvido no âmbito do Estudo do Risco Sísmico e Tsunamis do Algarve foi desenvolvida uma metodologia para quantificação de meios a alocar em caso de sismo, nomeadamente número de inspectores necessários para avaliação de estruturas e o volume
QuADro II - Valores médios de danos humanos e no parque edificado.

\begin{tabular}{|l|c|c|}
\hline \multicolumn{1}{|c|}{ Danos (valor médio) } & Carcavai, 6 & $\begin{array}{c}\text { SW Cabo } \\
\text { S. Vicente, 8 }\end{array}$ \\
\hline Edifícios a inspecionar & 8.817 & 49.149 \\
\hline $\begin{array}{l}\text { Tempo médio para } \\
\text { inspecções (dias) }\end{array}$ & 1.763 & 9.830 \\
\hline $\begin{array}{l}\text { No equipas inspecção/3 } \\
\text { mês inspecções }\end{array}$ & 29 & 164 \\
\hline $\begin{array}{l}\text { No técnicos/3 mês } \\
\text { inspecções }\end{array}$ & 59 & 328 \\
\hline
\end{tabular}

\begin{tabular}{|l|c|c|}
\hline \multicolumn{1}{|c|}{ Danos (valor médio) } & Carcavai, 6 & $\begin{array}{c}\text { SW Cabo } \\
\text { S. Vicente, 8 }\end{array}$ \\
\hline $\begin{array}{l}\text { Edifícios colapsados } \\
\text { (média) }\end{array}$ & 280 & 782 \\
\hline $\begin{array}{l}\text { Volume médio de } \\
\text { construção/edifício (m3) }\end{array}$ & \multicolumn{2}{|c|}{1350} \\
\hline $\begin{array}{l}\text { Estimativa de entulho } \\
\text { (m3) }\end{array}$ & 378.000 & 1.055 .700 \\
\hline
\end{tabular}

de entulho a remover. A aplicação desta metodologia revela-se de grande utilidade para a prossecução das actividades da protecção civil, nomadamente no que concerne à preparação para a resposta a eventos sísmicos de grande dimensão.

Tendo em consideração o número de técnicos necessários para a realização das inspecções, é fundamental planear este tipo de acções. As Ordens dos Engenheiros, Arquitectos, Engenheiros Técnicos e o LNEC são parceiros fundamentais nesta matéria.

\section{Referências bibliográficas}

Baptista, Maria Ana; Miranda, J. M.; Lima, Vânia; Catalão João; Roquette, Pedro (2008) - "Avaliação do Risco de Tsunami na Costa do Algarve". Instituto de Ciências da Terras e do Espaço.

Costa, E; Pires, P.; Vicêncio, H. (2010) - “Cenários de Avaliação de Danos”. Autoridade Nacional de Protecção Civil.

Gaspar, Jorge; Queirós, Margarida; Brito Henriques, Eduardo; Rodriguez, José; Palma, Pedro e Vaz, Teresa. (Novembro 2008) - "Caracterização e Estudo das Vulnerabilidades Humanas". Centro de Estudos Geográficos, Faculdade de Letras da Universidade de Lisboa.

LneC, Departamento de Estruturas, Núcleo de Engenharia Sísmica e Dinâmica de Estruturas (2008) - "Estudo do Risco Sísmico e de Tsunamis do Algarve (ERSTA). Relatório Final". Estudo do Risco Sísmico e de Tsunamis do Algarve. Laboratório Nacional de Engenharia Civil.

LouREnço, Cristina Isabel de Campos. (2007) - “Dissertação de Mestrado em Engenharia Civil: optimização de sistemas de demolição - demolição selectiva". Instituto Superior Técnico.

Mota dE SÁ, Francisco. SSA - Simulador sísmico do Algarve - Manual de Utilização. Relatório técnico WP22 realizado para o Estudo do Risco Sísmico e Tsunamis do Algarve. FUZZY. 